\title{
Magnetism of step-decorated Fe on Pd(110)
}

\author{
Dongqi Li, B. Roldan Cuenya,* J. Pearson, and S. D. Bader \\ Materials Science Division, Argonne National Laboratory, Argonne, Illinois 60439 \\ W. Keune \\ Laboratorium für Angewandte Physik, Gerhard-Mercator-Universität Duisburg, D-47048 Duisburg, Germany
}

(Received 7 March 2001; published 18 September 2001)

\begin{abstract}
We investigate the growth and magnetic properties of submonolayer Fe wedges on a stepped $\operatorname{Pd}(110)$ substrate with reflection high-energy electron diffraction (RHEED) and the surface magneto-optic Kerr effect. RHEED suggests that Fe atoms decorate the steps to form nanostripes. These stripes are ferromagnetic above 0.3 monolayer Fe coverage, or $\sim 6 \AA$ average stripe width, and have a magnetic easy axis along the surface normal. The onset temperatures of the broadened transition exhibit finite-size scaling with a shift exponent $\lambda$ of $1.2 \pm 0.3$, consistent with two-dimensional Ising expectations. The coercivity is less temperature dependent than the magnetization.
\end{abstract}

DOI: 10.1103/PhysRevB.64.144410

PACS number(s): 75.75.+a, 68.65.-k

Magnetic systems provide a rich testing ground to explore low-dimensional physics. An isolated one-dimensional (1D) Ising chain is known theoretically to exhibit no long-range magnetic order above zero temperature. In systems probed experimentally, many issues are of interest to address with respect to the existence of ferromagnetism at finite temperature, including the role of the magnetic anisotropy, finite-size effects, and nonequilibrium behavior. The fabrication of quasi-1D nanostriped systems presents a challenge where there has been real progress recently. Self-assembled, epitaxial monolayer (MD) stripes have been grown on stepped substrates by step decoration, ${ }^{1-5}$ with widths from one atomic row to $\sim 10^{2} \AA$. Elmers et al. ${ }^{3}$ demonstrated that Fe stripes on $\mathrm{W}(110)$ are ferromagnetic with an in-plane magnetic easy axis that is perpendicular to the stripes. Their finite-size scaling is in agreement with the 2D Ising model and extrapolates their experimental $T_{C}$ obtained from wires with width $\geqslant 44$ $\AA$ to a ferromagnetic onset of $8 \AA$, i.e., about four atomic rows. Furthermore, they present no unusual temperature or time dependence. In contrast, Shen et al. ${ }^{5}$ report strong temperature and time dependence in the remanent magnetization $M_{R}$ for Fe stripes consisting of connected islands at step edges on $\mathrm{Cu}(111)$. They find that $M_{R}$ is always significantly smaller than the saturation magnetization $M_{S}$ and report a small saturation field. Monte Carlo calculations on isolated stripes exhibit metastability with exceedingly long time constants compared to experimental time scales. ${ }^{6}$ It is important to explore additional systems to obtain a more complete view of the properties of such striped systems.

We investigate submonolayer Fe on stepped $\mathrm{Pd}(110)$. It is known that the initial growth of metals on $\operatorname{Pd}(110)$ surfaces is highly anisotropic and tends to form nanoscale stripes even on flat surfaces. ${ }^{7}$ By using a stepped $\operatorname{Pd}(110)$ substrate with the steps along the fast-diffusing direction, i.e., $\langle 1 \overline{1} 0\rangle,{ }^{7}$ smooth, straight $\mathrm{Fe}$ stripes are favored. In addition, Fe stripes are likely to retain their ferromagnetism on Pd since Pd is known to acquire an induced magnetic moment through proximity. ${ }^{8}$ The growth and magnetic properties of $\mathrm{Fe}$ on $\operatorname{Pd}(100)$ have been studied previously; the onset of ferromagnetism occurs at the island percolation limit. ${ }^{9}$ The magnetic easy axis is perpendicular to the film surface on $\mathrm{Fe} / \mathrm{Pd}(100)$ grown at low temperature and in plane for room-temperature growth. ${ }^{10}$ We recently studied the growth of $\mathrm{Fe}$ on vicinal $\operatorname{Pd}(110),{ }^{11}$ and found evidence for step decoration and therefore Fe stripe formation. We report in the present work magnetic properties of submonolayer Fe obtained via polar Kerreffect measurements. We find that the system exhibits ferromagnetic hysteresis with full remanence and perpendicular anisotropy even down to $\sim 0.3$ ML Fe coverage. The coverage dependence of the Curie temperature can be described by finite-size scaling. Below 0.7 ML, both $M_{R}$ and $M_{S}$ exhibit rapid, exponential decay with increasing temperature, in contrast to the behavior observed for 2D magnetic systems. The coercivity in the same coverage range exhibits weak temperature dependence, while the coercivity of the samples around or above $0.7 \mathrm{ML}$ is very sensitive to temperature.

The experiments were carried out in an ultrahigh vacuum (UHV) chamber equipped for the surface magneto-optic Kerr effect, reflection high-energy and low-energy electron diffraction (RHEED and LEED, respectively), Auger electron spectroscopy, and $e$-beam evaporators. The $\operatorname{Pd}(110)$ substrate possesses steps along $\langle 1 \overline{1} 0\rangle$ and an average terrace width of $\sim 20 \AA .{ }^{11}$ It was cleaned in UHV by cycles of sputtering and annealing. ${ }^{11}$ The resultant substrate and the subsequent $\mathrm{Fe}$ films are free of measurable contamination within the Auger sensitivity limit. Fe was evaporated at a rate of $0.2-0.3$ $\AA /$ min with the chamber pressure during deposition rising to $\sim(2-4) \times 10^{-10}$ Torr. Fe wedges were grown at $340 \mathrm{~K}$ with a slope of $\sim 0.2-0.4 \mathrm{ML} / \mathrm{mm}$. A thickness range of $0-6 \mathrm{ML}$ was covered but the present work focuses on the submonolayer region. Magnetic properties were studied in situ at 40$250 \mathrm{~K}$ along the wedges, mainly with the polar configuration of the Kerr effect.

Both the substrate and the Fe films are structurally ordered with sharp RHEED streaks, as shown in the inset of Fig. 1(a). The stepped structure is indicated by the intensity modulation along the $(0,0)$ streak, as seen in Fig. 1(b). The first Fe layer grows pseudomorphically with the same lateral lattice constant as the Pd. ${ }^{11}$ Figure 1(a) exhibits a set of RHEED oscillations taken at (i) the center and (ii) the tail of the $(0,0)$ streak, as marked in the inset. There are similar 


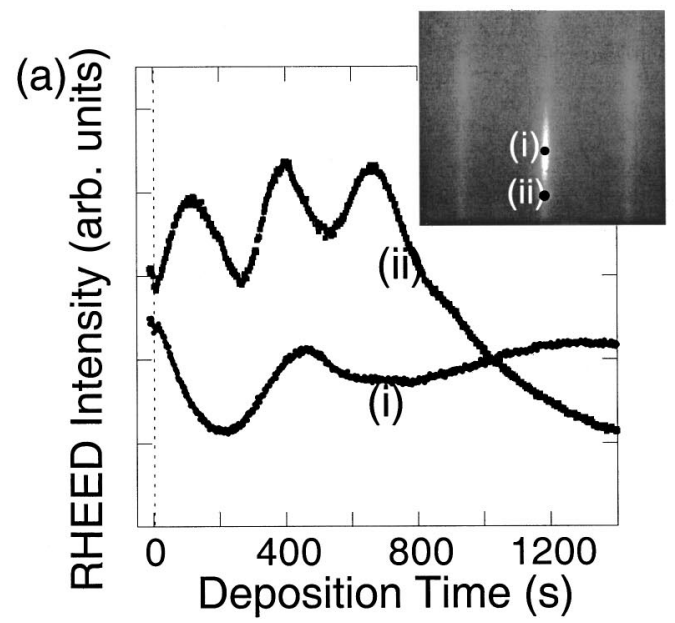

(b)

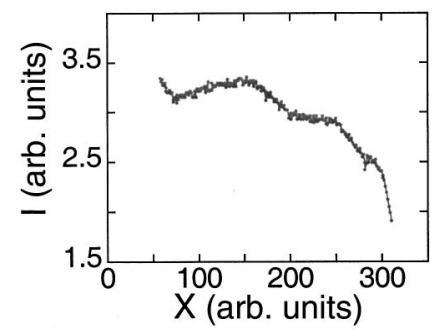

(c)

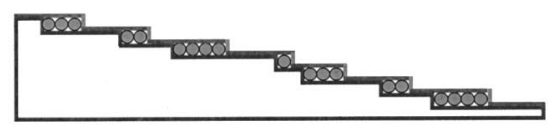

FIG. 1. (a) RHEED oscillations for Fe growth on stepped $\mathrm{Pd}(110)$ at $340 \mathrm{~K}$ observed at (i) the center of the $(0,0)$ streak; (ii) the low-angle tail of the $(0,0)$ streak. The inset shows a typical RHEED pattern, with the regions where the RHEED oscillations are taken indicated. The incident plane of the RHEED is in parallel with the Pd [001] direction and perpendicular to the steps. (b) Intensity modulation along the $(0,0)$ streak indicating the stepped surface (with $0.1 \mathrm{ML}$ of $\mathrm{Fe}$ ). (c) Schematics of the proposed stepdecoration growth mode. Note that at $0.5 \mathrm{ML}$ the terrace widths become significantly more uniform.

oscillations with $1 \mathrm{ML}$ period in the first $1-2 \mathrm{ML}$ at the center of the $(0,0)$ reflection, and on the $(1,0)$ and $(2,0)$ streaks (not shown). ${ }^{11}$ These observations are indicative of initial smooth, layer-by-layer growth. But (ii), taken at an exit angle of $\sim 1^{\circ}-2^{\circ}$, exhibits an oscillation with a half ML period up to $1.5 \mathrm{ML}$. We interpret the change in periodicity as due to Fe step decoration, ${ }^{11}$ as was first used by Gambardella et $a .^{2}$ to explain a $0.5 \mathrm{ML}$ peak in atom-beam scattering on stepped surfaces and confirmed by scanning tunneling microscopy (STM). Our Pd(110) is stepped with a relatively broad terrace-width distribution. To visualize a basis for the half-monolayer periodicity, imagine that $\mathrm{Fe}$ adatoms stay on the terrace they initially impinge on and diffuse to the step edges to form monolayer-high stripes. Then at any given Fe coverage, more atomic rows form on the wider terraces than on the narrower ones. At $\sim 0.5 \mathrm{ML}$, all terraces are half filled, giving rise to a narrowed distribution of terrace widths, as indicated in Fig. 1(c). The narrowing of the distribution sharpens the diffraction and yields the RHEED

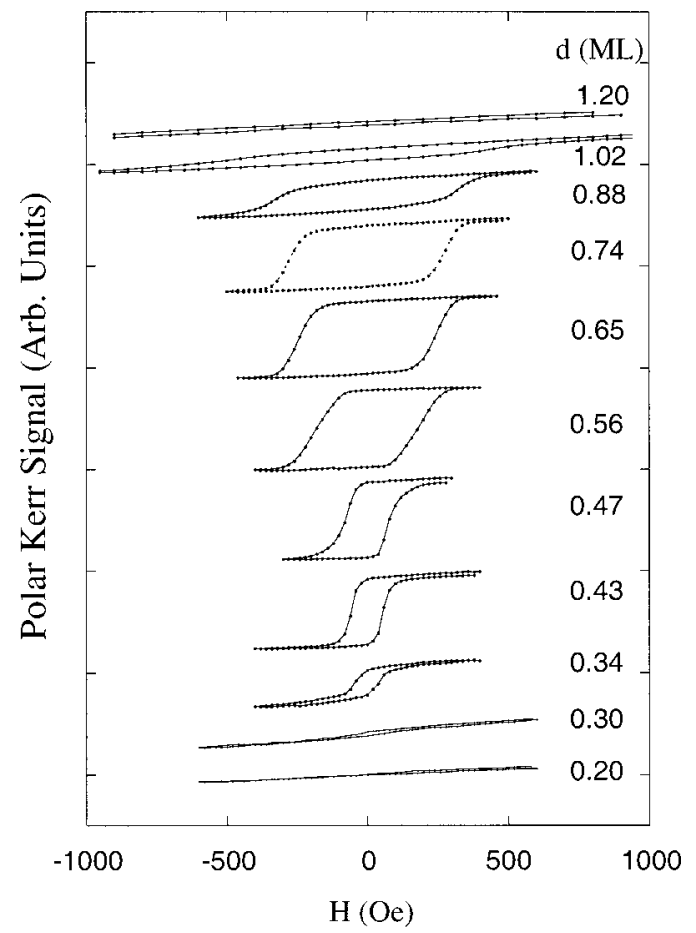

FIG. 2. Polar Kerr hysteresis loops of submonolayer Fe/Pd(110) at different coverages measured at $39 \mathrm{~K}$.

peak. Such a step-decoration growth hypothesis for $\mathrm{Fe}$ on $\operatorname{Pd}(110)$ will be further tested with UHV STM in the future.

Such results seem to be in disagreement with the previous understanding that the RHEED oscillations disappear when step-flow growth occurs. We believe that the difference stems from the ordered steps on the surface. RHEED probes the profile of the surface by interference of the electron beam. On a flat surface with occasional mosaic steps, the profile of the surface remains statistically the same during deposition, if the deposited atoms decorate the step edges. On a vicinal surface, however, the ordered stepped structure can be observed in RHEED as double peaks along the streak with their width and position determined by the lateral surface profile. ${ }^{12}$ The atoms attached to step edges may alter the staircase structure with a change in terrace-width distribution as discussed above, or a change in edge roughness. The RHEED intensity distribution, e.g., the widths of the peaks along the streak, should oscillate if the profile of a surface alters periodically during deposition, with a period that may or may not correspond to $1 \mathrm{ML}$. Indeed, similar intensity oscillations have been observed with atom-beam scattering and confirmed as step-decoration growth with STM. ${ }^{2}$

Figure 2 illustrates representative magnetic hysteresis loops at different Fe coverages, measured at $39 \mathrm{~K}$, with the polar Kerr effect. The onset of ferromagnetic ordering is at $0.3 \mathrm{ML}$, compared to $0.5-1 \mathrm{ML}$ for $\mathrm{Fe} / \mathrm{Pd}(100)$ where island percolation occurs. ${ }^{9}$ All the loops exhibit full remanence. No time dependence is observed on our experimental time scale. (The linear background slope of the loops is an artifact from the window or paramagnetic impurities in the substrate.) The polar $M_{R}$ initially increases with $\mathrm{Fe}$ coverage, reaches a maximum at $\sim 0.6-0.7 \mathrm{ML}$, and then decreases while the 
coercivity $H_{C}$ increases. This decrease in $M_{R}$ is accompanied by the emergence of a longitudinal Kerr signal. This indicates that the easy axis is perpendicular to the surface up to $0.6-0.7 \mathrm{ML}$. Hence, a thickness of $\sim 0.6-0.7 \mathrm{ML}$ is likely where interstripe interactions emerge or the individual stripes start to connect to alter the magnetic anisotropy. The initial perpendicular anisotropy may be induced by the step edges and/or the terraces. For a 1D Co chain on Pd(110), tightbinding calculations predict an out-of-chain easy axis, ${ }^{13}$ while a free-standing chain has an in-chain anisotropy. In $\mathrm{Fe} / \mathrm{Pd}(001)$ experiments, the easy axis is in plane for $300 \mathrm{~K}$ growth and perpendicular to the surface for $100 \mathrm{~K}$ growth. ${ }^{10}$ $\mathrm{Fe}$ stripes on $\mathrm{Cu}(111)$ exhibit perpendicular anisotropy, as does an $\mathrm{Fe}$ monolayer on $\mathrm{Cu}(111),{ }^{4}$ while $\mathrm{Fe}$ stripes on $\mathrm{W}(110)$ have an in-plane easy axis that is perpendicular to the stripes. ${ }^{3}$

Figure 3 shows loops at different temperatures for 0.5 and 0.7 ML. $M_{R}$ tracks $M_{S}$ within experimental error at virtually all temperatures. This is different from the case of Fe stripes on $\mathrm{Cu}(111)$, where $M_{R}$ is always significantly less than $M_{S}$, especially at higher temperatures. At $0.7 \mathrm{ML}$, both $M_{R}$ and $H_{C}$ decrease as temperature increases. At 0.5 ML, however, while $M_{R}$ decreases rapidly, most significantly, $H_{C}$ decreases only gradually and does not approach zero, as shown more clearly in the Fig. 4 inset. All the samples with a coverage less than 0.5 ML show the same characteristics behavior. Ordinarily, $H_{C}$ should be strongly temperature dependent and approach zero at high temperature. ${ }^{14}$ However, the nucleation process or step pinning could significantly alter the magnetic switching process and hence the coercivity in stripe systems. It would be interesting to understand this behavior and to see if it is related to the quasi-1D geometry.

The temperature dependence of $M_{R}$ and $H_{C}$ is replotted in Fig. 4 from the data in Fig. 3. At $0.9 \mathrm{ML}$, the $M_{R}$ curve behaves like the order parameter in a second-order phase transition. At $0.5 \mathrm{ML}$, however, $M_{R}$ decreases exponentially throughout the temperature range studied. No inflection point, a common way to define $T_{C}$, exists in a wide temperature range of $\sim 40-120 \mathrm{~K}$. For $0.7 \mathrm{ML}$, two distinctly different behaviors are evident below and above $\sim 100 \mathrm{~K}$, which likely relates to the change in magnetic anisotropy mentioned above. All of the curves are reversible upon thermal cycling. The temperature dependence we observe is different from that reported for Fe stripes on $\mathrm{W}(110)$, where 2D Ising behavior was observed. It is also different from the case of Fe stripes on $\mathrm{Cu}(111)$, where $M_{R}$ decays while $M_{S}$ follows 2D Ising expectations.

An exponential-type decay in both $M_{R}$ and $M_{S}$ has not been reported previously for such ferromagnetic systems to our knowledge, quasi-1D or not, although superparamagnetic systems indeed show a similar behavior in $M_{R}$. We can rule out superparamagnetism as the origin for the temperature dependence we observe. In superparamagnetic systems, blocks of spins behave like giant magnetic moments that rotate in direction in a temperature-activated process for $M_{R}$ above the blocking temperature $T_{B}$. Even at $0 \mathrm{~K}, M_{R}$ should attain a value of $M_{S} / 2$ after field cooling and subsequent removal of the field, ${ }^{15}$ while $M_{S}$ exhibits Ising behavior. ${ }^{15}$ These properties are different from our case, where $M_{R}$ and $M_{S}$ behave similarly. In addition, our measured $H_{C}(T)$ and
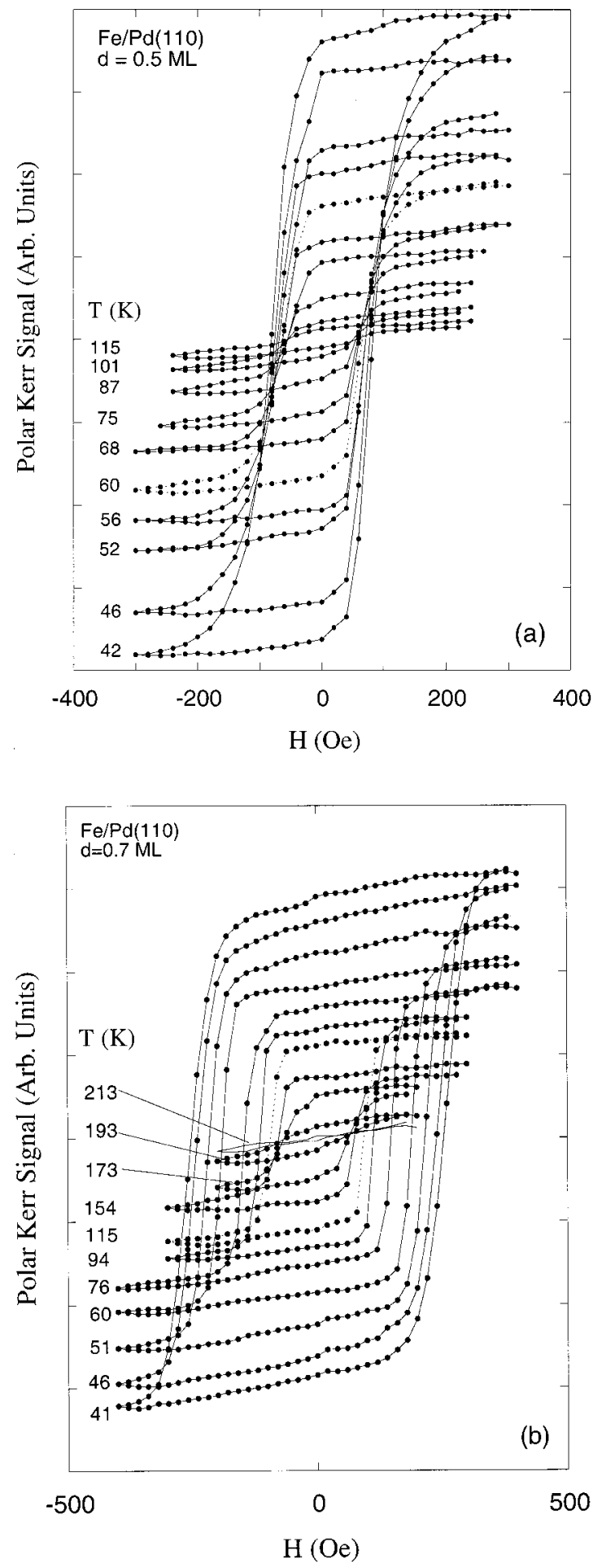

FIG. 3. Polar Kerr loops at different temperatures for (a) 0.5 and (b) $0.7 \mathrm{ML} \mathrm{Fe}$.

$M(H, T)$ do not follow expectations for superparamagnetic systems. $^{15}$

Although $T_{C}$ is ill defined at low coverage, we adopt an operational definition for an onset temperature $T_{C}^{*}$ as illustrated in the inset of Fig. 5. At each temperature, $M_{R}$ is measured across a wedge and then linearly extrapolated to zero at low coverage to determine a critical coverage. The 


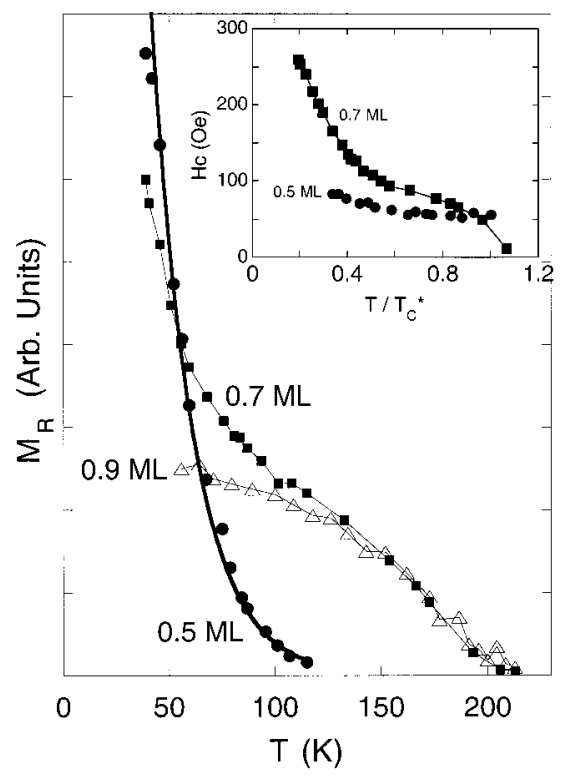

FIG. 4. Remanent magnetization $M_{R}$ vs temperature at different coverages. The solid line for $0.5 \mathrm{ML}$ is an exponential fit to the data, while the other lines are guides to the eye. The inset shows the coercivity $H_{C}$ vs $T / T_{C}^{*}$ for 0.5 and $0.7 \mathrm{ML}$, where $T_{C}^{*}$ is defined in Fig. 5.

measurement temperature is then defined as the $T_{C}^{*}$ at that critical coverage. As shown in Fig. $5, T_{C}^{*}$ increases as a function of coverage, or stripe width. (Only the data from below $0.7 \mathrm{ML}$ are plotted since the magnetic behavior changes beyond that point.) Using $T_{C}^{*}$ to approximate $T_{C}$, the initial coverage dependence can be described by the finite-size scaling expression

$$
T_{C}(d)=T_{C 0}\left[1-\left(d / d_{0}\right)^{-\lambda}\right],
$$

where $d$ is the coverage and $\lambda$ is the shift exponent. ${ }^{16}$ The value of $d_{0}$ is found from the fitting to be $0.32 \pm 0.09 \mathrm{ML}$,

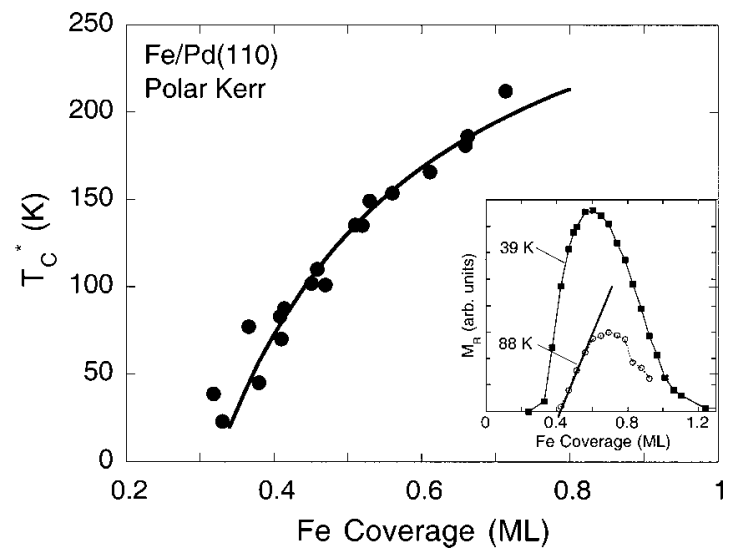

FIG. 5. The effective $T_{C}^{*}$ vs Fe coverage. The solid line is a finite-size-scaling fit, as discussed in the text. The value of the shift exponent is $\lambda=1.2 \pm 0.3$, consistent with $\lambda=1$ for the $2 \mathrm{D}$ Ising model. The inset shows the operational definition of the onset temperature $T_{C}^{*}$ at each coverage: $M_{R}$ across a wedge is extrapolated to zero at low coverage to obtain the critical coverage associated with the given measurement temperature to determine $T_{C}^{*}$. below which the system is no longer ferromagnetic. For an average terrace width of $W \sim 20 \AA$, this would correspond to a stripe width of $w_{0}=W d_{0} /(1 \mathrm{ML}) \sim 6 \AA . \quad \lambda=1.2 \pm 0.3$, consistent with the 2D Ising model, which yields $\lambda=1$ as $2 \mathrm{D}$ stripes change their width. ${ }^{16}$ The observation of finite-size scaling suggests that the stripes are effectively isolated. Similar behavior for $T_{C}(d)$ was observed by Elmers et al. ${ }^{3}$ on $\mathrm{Fe} / \mathrm{W}(110)$ for stripes with widths $\geqslant 44 \AA$. The differences in the width range of the stripes may explain the differences in temperature dependence of $M_{R}$ between their work and ours. The narrower Fe stripe arrays on stepped W(110) exhibited interstripe dipolar couplings, which tend to align the moments in plane. ${ }^{4}$ For $\mathrm{Fe} / \mathrm{Pd}(110)$, since the easy axis is perpendicular to the surface, a dipolar interaction would cause antiferromagnetic alignment among the stripes. No sign of such a coupling is observed, given the full remanence, which may result from the strong perpendicular anisotropy.

In addition to the $T_{C}$ shift effect, it is also anticipated that $T_{C}$ for a finite system should be rounded, such that

$$
\delta=\frac{\Delta T}{T_{C 0}} \propto 1 / d^{\theta},
$$

where $\Delta T$ is due to the fact that the correlation length cannot diverge in a finite system. ${ }^{16}$ For a finite-size 2D Ising system, $\theta=1$, indicating a significant broadening in the transition region as $d$ decreases. ${ }^{16}$ This may be the origin of our unusual $M(T)$ behavior, although measurements to lower temperatures would provide valuable confirmation of this idea. A distribution of stripe widths, and therefore $T_{C}$ values, should also cause a broadening of the transition region, ${ }^{17}$ but the experimental $M(T)$ cannot be described by a Gaussian distribution of $T_{C}$ values each with a $2 \mathrm{D}$ temperature dependence.

In summary, we have grown $\mathrm{Fe}$ wedges onto a vicinal $\operatorname{Pd}(110)$ surface at $340 \mathrm{~K}$ and find evidence that the $\mathrm{Fe}$ atoms decorate the steps and form pseudomorphic monolayer stripes at submonolayer coverage up to $\sim 0.7 \mathrm{ML}$. The onset of ferromagnetic ordering is at $\sim 0.3 \mathrm{ML}$, which is below the limit expected for percolation. The stripes initially have a magnetic easy axis perpendicular to the surface, but above $\sim 0.7 \mathrm{ML}$ the easy axis starts to reorient. Both $M_{R}$ and $M_{S}$ behave similarly and decay exponentially on warming over an extended temperature range of $\sim 40-100 \mathrm{~K}$. While superparamagnetism can be ruled out, the finite-size rounding of the Curie temperature of quasi-1D stripes may underlie the result. In this regard, it is interesting to note that the behavior of the effective Curie temperature as a function of coverage is in accordance with scaling expectations for a finite -size 2D Ising system. The coercivity shows intriguing behavior at $\sim 0.5$ ML Fe coverage, suggesting that nucleation of reversed domains in stripes or step pinning may profoundly influence the magnetization switching process.

We thank A. Berger, M. Farle, and H. J. Elmers for helpful discussions. This work was supported by the U.S. Department of Energy, Office of Science, Basic Energy SciencesMaterials Sciences, under Argonne Contract No. W-31-109ENG-38 and by the Deutsche Forschungsgemeinschaft (GRK 277). 
*Permanent address: Laboratorium für Angewandte Physik, Gerhard-Mercator-Universität Duisburg, D-47048 Duisburg, Germany.

${ }^{1}$ F. J. Himpsel, T. Jung, A. Kirakosian, J.-L. Lin, D. Y. Petrovykh, H. Rauscher, and J. Viernow, MRS Bull. 24(8), 20 (1999); F. J. Himpsel, T. Jung, and J. E. Ortega, Surf. Rev. Lett. 4, 371 (1997).

${ }^{2}$ P. Gambardella, M. Blanc, H. Brune, K. Kuhnke, and K. Kern, Phys. Rev. B 61, 2254 (2000); P. Gambardella, M. Blanc, L. Bürgi, K. Kuhnke, and K. Kern, Surf. Sci. 449, 93 (2000).

${ }^{3}$ H. J. Elmers, J. Hauschild, H. Höche, U. Gradmann, H. Bethge, D. Heuer, and U. Köhler, Phys. Rev. Lett. 73, 898 (1994).

${ }^{4}$ J. Hauschild, H. J. Elmers, and U. Gradmann, Phys. Rev. B 57, R677 (1998).

${ }^{5}$ J. Shen, R. Skomski, M. Klaua, H. Jenniches, S. Sundar Manoharan, and J. Kirschner, Phys. Rev. B 56, 2340 (1997); J. Shen, M. Klaua, P. Ohresser, H. Jenniches, J. Barthel, C. V. Mohan, and J. Kirschner, ibid. 56, 11134 (1997).

${ }^{6}$ D. Wingert and D. Stauffer, Physica A 219, 135 (1995); P. Sen, D. Stauffer, and U. Gradmann, ibid. 245, 361 (1997).

${ }^{7}$ J.-P. Bucher, E. Hahn, P. Fernandez, C. Massobrio, and K. Kern,
Europhys. Lett. 27, 473 (1994).

${ }^{8}$ H. J. Choi, R. K. Kawakami, E. J. Escorcia-Aparicio, Z. Q. Qiu, J. Pearson, J. S. Jiang, Dongqi Li, and S. D. Bader, Phys. Rev. Lett. 82, 1947 (1999), and references therein.

${ }^{9}$ X. F. Jin, J. Barthel, J. Shen, S. S. Manoharan, and J. Kirschner, Phys. Rev. B 60, 11809 (1999).

${ }^{10}$ C. Liu and S. D. Bader, J. Appl. Phys. 67, 5758 (1990).

${ }^{11}$ B. Roldan Cuenya, J. Pearson, Chengtao Yu, Dongqi Li, and S. D. Bader, J. Vac. Sci. Technol. A 19, 1182 (2001).

${ }^{12}$ D. Saloner, J. A. Martin, M. C. Tringides, E. E. Savage, C. E. Aumann, and M. G. Lagally, J. Appl. Phys. 61, 2884 (1987).

${ }^{13}$ J. Dorantes-Dávila and G. M. Pastor, Phys. Rev. Lett. 81, 208 (1998).

${ }^{14}$ H. Kronmüller, K.-D. Durst, and M. Sagawa, J. Magn. Magn. Mater. 74, 291 (1988).

${ }^{15}$ C. L. Chien, J. Appl. Phys. 69, 5267 (1991), and references therein.

${ }^{16}$ M. E. Fisher and A. E. Ferdinand, Phys. Rev. Lett. 19, 169 (1967); M. E. Fisher and M. N. Barber, ibid. 28, 1516 (1972)

${ }^{17}$ H.-J. Elmers, J. Hauschild, and U. Gradmann, Phys. Rev. B 54, 15224 (1996). 